\title{
Teorías de la referencia, filosofía experimental y calibración de intuiciones*
}

\author{
(Theories of Reference, Experimental Philosophy, and the Calibration of Intuitions)
}

\author{
Manuel Pérez Otero
}

Received: $26 / 11 / 2015$

Final Version: 21/06/2016

BIBLID 0495-4548(2017)32:1p.41-62

DOI: $10.1387 /$ theoria. 15463

RESUMEN: E. Machery y algunos colaboradores suyos han utilizado datos sobre encuestas para criticar el anti-descriptivismo sobre los nombres propios de Kripke. Presento aquí diversos inconvenientes de esas encuestas. Algunas de mis objeciones conciernen a su ambigüedad. En particular, las respuestas que — según Machery et al.- revelan intuiciones descriptivistas pueden interpretarse como respuestas anti-descriptivistas, por razones que - por lo que yo sé- nadie ha destacado hasta ahora. Además, los casos presentados a los encuestados son aparentemente inconsistentes. También abordo otras cuestiones relacionadas con el papel de las intuiciones en la filosofía; las tesis de Machery et al. dependen de un supuesto injustificado: no existen capacidades expertas respecto a las intuiciones.

Palabras clave: Kripke; Machery; descriptivismo; capacidades expertas; nombres propios; semántica inter-cultural; experimentos mentales; justificación empírica.

ABSTRACT: E. Machery and some collaborators have used survey data to criticize Kripke's anti-descriptivism about proper names. I highlight a number of drawbacks in the tests of Machery et al. Some of my objections concern their ambiguity. In particular, the responses that — according to them - reveal descriptivist intuitions can be interpreted as anti-descriptivist responses, for reasons that — as far as I know- have not been pointed out so far. Furthermore, their vignettes are apparently inconsistent. I also discuss other issues related to the role of intuitions in philosophy; Machery et al.'s theses depend on an unjustified assumption: there is not expertise regarding intuitions.

Keywords: Kripke; Machery; descriptivism; expertise; proper names; cross-cultural semantics; thought experiments; empirical justification.

\section{Introducción}

El descriptivismo sobre la referencia de los nombres propios propone que un hablante asocia con cada nombre propio del lenguaje el material descriptivo correspondiente a una o varias descripciones definidas; además el referente o denotación del nombre es el único individuo que satisface dicha descripción. Hace casi medio siglo, esa concepción descriptivista

* Algunos de los contenidos de este artículo se han presentado en una conferencia invitada (Universidad de Granada, Marzo 2016) y en el $2^{\text {nd }}$ Portuguese Congress of Philosophy (Oporto, Septiembre 2016). Agradezco sus comentarios a los participantes en esos actos, así como a Agustín Vicente, que leyó versiones preliminares de estas ideas y me envió diversas observaciones. Financiación: Proyecto «Objetividad-subjetividad en el conocimiento y en la representación singular» (FFI2015-63892-P), (MINECO/FEDER, UE). / Grupo de investigación consolidado LOGOS (2014SGR81), AGAUR. 
fue objeto de diversas críticas por parte de Donnellan, Kaplan, Kripke, Putnam y otros filósofos del lenguaje. Entre los trabajos fundamentales de ese movimiento anti-descriptivista destaca Naming and Necessity (Kripke 1972/1980). Una de las argumentaciones importantes contra el descriptivismo desarrolladas por Kripke invocaba una situación imaginaria: el caso Gödel-Schmidt. Ese caso y otros experimentos mentales en la misma línea pondrían de manifiesto que muchos de nuestros nombres propios no tienen como referente lo que establecería la teoría descriptivista, al menos en su versión originaria, defendida por Frege y por Russell. Conforme a una interpretación habitual bastante natural del caso Gödel-Schmidt, la supuesta justificación anti-descriptivista que de él se derivaría concierne a nuestras intuiciones semánticas, que —en su mayoría - serían inconsistentes con la teoría descriptivista. Si es así, y como es usual en las propuestas filosóficas, Kripke, al evaluar qué podemos aprender del caso Gödel-Schmidt, habría apelado implícitamente a sus propias intuiciones semánticas, asumiendo que son mayoritariamente compartidas por quienes reflexionamos y juzgamos también sobre ese caso.

En años recientes, algunos autores defienden tesis diversas sobre teorías filosóficas y sobre las intuiciones que las respaldarían, basándose en lo que se ha denominado «filosofía experimental»: a grandes rasgos, hipótesis acerca de las intuiciones de la gente, inspiradas por estudios estadísticos diseñados para detectar tales intuiciones. Uno de los practicantes de esa filosofía «experimental», E. Machery, junto con diversos colaboradores, ha elaborado algunos cuestionarios destinados a poner en duda la crítica al descriptivismo procedente del caso Gödel-Schmidt (cf. Machery et al. 2004; Machery et al. 2015). Concretamente, E. Machery y sus colaboradores rechazan que las intuiciones semánticas anti-descriptivistas tengan la generalidad apropiada para constituir una buena justificación. Dichas intuiciones serían minoritarias — sostienen- en algunas comunidades; por ejemplo, en grupos de universitarios asiáticos por ellos encuestados.

El objetivo principal del presente trabajo es contribuir a clarificar las diversas deficiencias de que adolecen los trabajos mencionados, incluyendo una versión reciente: Machery et al. (2015). Varias objeciones ya formuladas en la literatura inciden en el hecho de que las encuestas elaboradas por Machery et al. resultaban ambiguas, y no permitían una interpretación clara de las respuestas obtenidas (cf. Ludwig 2007; Deutsch 2009). Precisamente, una motivación de las nuevas encuestas descritas en Machery et al. (2015) era minimizar ese problema. El grueso de mis objeciones también se vincula a la patente equivocidad de los cuestionarios utilizados por Machery et al., incluyendo los de 2015. Entre esas objeciones, señalaré algunos elementos novedosos que no han sido mencionados por ningún comentarista, o a los que no se les ha otorgado la importancia que tienen (secciones 3, 4 y 6). Por ejemplo, las situaciones presentadas a los sujetos encuestados parecen inconsistentes (sec. 3), debido al uso del verbo 'aprender' [learn] por parte de Machery et al. Es algo que habían apuntado otros autores, pero no se había perfilado con suficiente nitidez en qué consiste la crítica. Además - y de mayor importancia-, las respuestas supuestamente descriptivistas de los sujetos pueden interpretarse como respuestas anti-descriptivistas (sec. 4); sólo un autor, Kallestrup (2016), sostendría una tesis similar, pero basándose en cosideraciones muy diferentes a las mías. También evaluaré muy brevemente algunos aspectos de otras críticas que se han realizado (cf. Martí 2009; Deutsch 2009), lo cual nos conducirá a reflexionar sobre el papel de las intuiciones en la justificación de las teorías filosóficas (secs. 6, 7 y 8 ). 


\section{El caso Gödel-Schmidt}

La concepción descriptivista sobre la referencia de los nombres propios puede dar lugar a teorías diversas, dependiendo de la posición mantenida respecto a ciertos parámetros. Voy a centrarme en las dos versiones evaluadas por Kripke cuando considera el caso Gödel-Schmidt. Según el descriptivismo monista, de Frege, Russell, Carnap, Quine y otros, el vínculo semántico se da entre el nombre y una única descripción. Kripke (1972/1980) combate también un descriptivismo pluralista, representado por Searle, en el cual el nombre se vincula con muchas descripciones; ${ }^{1}$ incluso afirma que el resultado de nuestras reflexiones sobre el caso Gödel-Schmidt sería también válido - variando concomitantemente el ejemplo- contra dicho descriptivismo pluralista; pero esa afirmación puede ser controvertida, y - en cualquier caso- las encuestas presentadas por Machery et al. conciernen a un descriptivismo monista, que será el que aquí nos ocupará.

El tipo de descripción asociada al nombre que Frege y Russell tenían en mente expresa alguna propiedad o relación identificadora del referente ajena a cuestiones lingüísticas o metalingüísticas; se dice a veces que el referente se identifica por sus «hazañas» o «actos» [deeds] (el autor de La Metafísica, el hombre que denunció a Catilina, etc.), aunque —naturalmente - algunas de esas condiciones no conciernen a ninguna acción realizada por el referente (el hombre denunciado por Cicerón, el rey de Francia, el mayor planeta del sistema solar). Versiones sofisticadas del descriptivismo monista proponen que asociamos el nombre con alguna propiedad compleja en la cual intervienen conceptos directa o indirectamente semánticos: el individuo que está en el origen de la cadena causal correspondiente al nombre, o el individuo nombrado por los usos del nombre, o algo similar. Tanto el caso Gödel-Schmidt imaginado por Kripke como el caso, o los casos, Gödel-Schmidt-John imaginado(s) por Machery et al. versan sobre el descriptivismo clásico del primer tipo, no sobre esas otras versiones sofisticadas, que - por tanto- dejaremos de lado.

Kripke señala una distinción adicional entre dos versiones del descriptivismo. En la versión fuerte, la descripción da el significado del nombre, en el sentido de ser semánticamente equivalente al mismo; nombre y descripción son sinónimos. La versión débil no se compromete con dicha equivalencia, sólo sostiene que la descripción fija la referencia del nombre. ${ }^{2}$ Las dos teorías descriptivistas que centrarán nuestra atención son el descriptivismo monista de «hazañas» en sus versiones débil y fuerte. En adelante, y para simplificar, cuando hable aquí de descriptivismo me referiré a alguna de esas dos versiones.

Conforme al descriptivismo, asociamos semánticamente con un nombre propio una cierta descripción definida. Kripke nos invita a suponer que para el nombre 'Gödel' esa descripción es 'el descubridor de la incompletud de la aritmética'. Es claro que nada crucial depende de este caso particular; si el filósofo descriptivista fuera reticente ante este ejemplo, podría escoger él mismo uno alternativo: 'Fleming' y 'el descubridor de la penicilina', pongamos por caso, o algún otro par de expresiones. El descriptivismo implica dos subtesis:

1 Así, conforme al descriptivismo monista, cada hablante asocia con un nombre una determinada expresión de la forma 'el/la F'. Según el descriptivismo pluralista, el hablante asocia con el nombre una pluralidad de expresiones de esa forma.

2 En las secciones 4 y 5 examinaremos la relevancia de esta distinción con respecto a la discusión principal sobre los diseños experimentales de Machery et al. En Machery et al. (2015, nota 5) aseveran que toda su discusión conciernen al descriptivismo débil, sobre la determinación de la referencia. 
(i) Para todo $x$, si $x$ es el descubridor de la incompletud de la aritmética, entonces 'Gödel' nombra (refiere) a $x$

(ii) Para todo $x$, si 'Gödel' nombra (refiere) a $x$, entonces $x$ es el descubridor de la incompletud de la aritmética

Supongamos, no obstante, que hubiera sido otro individuo, Schmidt, quien descubrió la incompletud de la aritmética; supongamos que Gödel se apropió secretamente del manuscrito, se atribuyó el mérito y se deshizo de Schmidt. El descriptivismo implica que en tales circunstancias:

a) 'Gödel' nombraría (referiría) a Schmidt (por (i), pues Schmidt habría descubierto la incompletud de la aritmética)

b) 'Gödel' no nombraría (referiría) a ese sujeto que se apropió secretamente del manuscrito (por (ii), pues dicho sujeto no habría descubierto la incompletud de la aritmética)

Según Kripke, ambas tesis, (a) y (b), son falsas. Aunque no lo explicita, parece basarse en que nuestras intuiciones son claramente contrarias a cada una de esas dos tesis (cf. nuestras secciones 7, 8 y 9); si es así, nuestras intuiciones ante ejemplos como el caso Gödel-Schmidt son anti-descriptivistas.

\section{El caso Gödel-Schmidt-John}

Machery et al. (2004) contiene varias consideraciones contrarias a la posición anti-descriptivista desarrollada en Kripke (1972/1980); algunas de ellas —en caso de ser correctas- serían de largo alcance. Por ejemplo, expresan dudas generales sobre lo que ellos consideran que es la metodología filosófica usual, «de sillón», ilustrada por Kripke y otros autores. O también, más concretamente, los resultados de sus encuestas son presentados como si constituyeran indicios importantes para rechazar, en general, los argumentos anti-descriptivistas de Kripke. Sin embargo, esas opiniones están basadas en estudios estadísticos acerca del caso Gödel-Schmidt y acerca de otro muy similar, relativo al nombre 'Jonás'. Como ya se ha señalado en la literatura (cf., por ejemplo, Ichikawa et al. 2012), el caso Gödel-Schmidt y el caso sobre 'Jonás' son sólo un componente entre muchos otros de las reflexiones de Kripke. Incluso si los estudios de Machery et al. hubieran sido exitosos (un objetivo central de este trabajo es mostrar que no han sido exitosos), eso todavía quedaría muy lejos de constituir una refutación de «los argumentos anti-descriptivistas » de Kripke.

No voy a desarrollar aquí críticas a los aspectos más ambiciosas de las sugerencias y opiniones de Machery et al. Me centraré en dos hipótesis más modestas que, aparentemente, quedarían apropiadamente respaldadas por sus estudios estadísticos:

(M1) casos del tipo Gödel-Schmidt no respaldan el rechazo del descriptivismo; en particular,

(M2) las supuestas intuiciones anti-descriptivistas puestas de relieve en casos del tipo Gödel-Schmidt no son suficientemente generales (para constituir indicios contrarios al descriptivismo). 
Como intentaré mostrar en las próximas secciones, los experimentos aportados por Machery et al. distan mucho de justificar las tesis (M1) y (M2). Bajo mi punto de vista, ninguna de las dos tesis es verdadera. Pero lo relevante aquí, es examinar si los experimentos de Machery et al. permitirían respaldarlas. Para empezar, veamos el primero de esos diseños experimentales.

En Machery et al. (2004) se exponen los resultados de encuestas realizadas a diversos grupos de sujetos a quienes se les presentó una situación muy cercana al caso GödelSchmidt: el caso Gödel-Schmidt-John. Se les relató lo siguiente. Un individuo, John, ha aprendido [has learned] que Gödel es el hombre que descubrió la incompletud de la aritmética; eso es lo único que John ha oido sobre Gödel, aunque tiene conocimientos técnicos suficientes para enunciar qué dice dicho teorema de incompletud. Pero supongamos que fue otra persona, cuyo nombre es 'Schmidt', quien descubrió la incompletud de la aritmética; Gödel se apropió secretamente del manuscrito, se atribuyó el mérito y se deshizo de Schmidt. Desde entonces Gödel ha sido conocido [has been known] como el hombre que descubrió la incompletud de la aritmética. Se pidió entonces a los encuestados que eligieran entre A y B como respuesta a la pregunta «¿de quién habla [talks about] John cuando usa el nombre 'Gödel'?»:

A: de la persona que realmente descubrió la incompletud de la aritmética

B: de la persona que se apropió del manuscrito y se atribuyó el mérito

Un grupo de sujetos encuestados lo formaban estudiantes universitarios estadounidenses. Otro grupo lo formaban estudiantes asiáticos de la Universidad de Hong Kong, hablantes del inglés, pero cuya lengua materna es el chino. La prueba se presentó enteramente en inglés. Los resultados fueron los siguientes. En el grupo de estadounidenses, el $42 \%$ respondió A, el 58\% respondió B. En el grupo de asiáticos, el $71 \%$ respondió A, el 29\% respondió $\mathrm{B}$.

Machery et al. (2004) presuponen que la respuesta A es una respuesta descriptivista (en la sección 4 plantearé dudas ante esa presuposición) y la respuesta B es una respuesta antidescriptivista, consistente con la teoría histórico-causal de la referencia de los nombres, defendida - entre otros- por Kripke. ${ }^{3}$ Siendo así, interpretan estos resultados como datos claros favorables a sus tesis (M2) y (M1).

Incluso destacan la variabilidad de respuestas en cada grupo: el mero hecho de que haya un $42 \%$ de estadounidenses encuestados que respondan en consonancia con el descriptivismo podría prestar cierto apoyo — dan a entender - a las tesis (M2) y (M1) (cf. Machery et al. 2004, B8). Pero si tienen eso en mente, están interpretando de forma poco caritativa las apelaciones -implícitas o explícitas - a las intuiciones por parte de los filósofos. Cuando un filósofo sugiere o afirma que nuestras intuiciones son favorables a cierta tesis

3 Según dicha teoría histórico-causal de la referencia: el primer usuario del nombre fija su referencia ostensivamente o mediante una descripción definida; los otros usuarios están conectados con él a través de una «cadena» en que cada «eslabón» intenta usar el nombre con la misma referencia con que lo usa la persona de quien lo recibe; típicamente, en virtud de esa cadena causal denota un nombre a su referente. Machery et al. describen la problemática como un contraste entre la teoría descriptivista y la teoría histórico-causal. No obstante, la respuesta B es una respuesta anti-descriptivista, compatible no sólo con la teoría histórico-causal, sino también con otras posibles versiones del anti-descriptivismo. Por eso prefiero presentar la problemática como un contraste entre descriptivismo y anti-descriptivismo, de forma que no necesitamos profundizar en las características de la teoría histórico-causal. 
no pretende, normalmente, que exista unanimidad; no pretende que el $100 \%$ de los sujetos respondan en consonancia con tales intuiciones. Ciertamente, las discrepancias requerirán algún tipo de explicación. Pero, en términos generales, una amplia mayoría de intuiciones favorables a cierta tesis, $T$, constituyen indicio claro favorable a $T$; un indicio que puede apropiadamente describirse mediante aseveraciones como: nuestras intuiciones indican que Tes verdad. (Si tenemos en cuenta los problemas de los cuestionarios sobre los casos GödelSchmidt-John — que expondré en las siguientes secciones-, así como la distinción entre intuiciones expertas e intuiciones comparativamente no tan expertas - cf. la sección 9todavía resulta menos importante ese $42 \%$ de estadounidenses encuestados que supuestamente dieron respuestas descriptivistas.) En adelante, restringiré la discusión a los otros datos que — de forma más nítida - aparentemente permitirían aceptar las tesis (M2) y (M1): las respuestas supuestamente descriptivistas de una mayoría (71\%) de sujetos del grupo de estudiantes asiáticos.

Antes de centrarnos en los casos Gödel-Schmidt y Gödel-Schmidt-John, resalto otro problema de Machery et al. (2004) (cf. también Ludwig 2007, p. 141; Sytsma y Livengood 2011, p. 318). Los datos experimentales sobre el caso Gödel-Schmidt-John —según los interpretan Machery et al.- - señalarían que el grupo de estudiantes asiáticos tienen intuiciones mayoritariamente descriptivistas. Sin embargo, los datos obtenidos en pruebas similares que ellos mismos realizaron, también presentados en esa publicación, concernientes al nombre 'Jonás' indicarían justo lo contrario; indicarían que los estudiantes asiáticos tienen intuiciones mayoritariamente anti-descriptivistas. Así pues, incluso si los estudios sobre el caso Gödel-Schmidt-John hubieran sido exitosos, ${ }^{4}$ no podría legítimamente afirmarse que los datos experimentales presentados en ese trabajo respaldan la hipótesis de que los estudiantes asiáticos encuestados tienen intuiciones mayoritariamente descriptivistas.

\section{Equivocidad e inconsistencia en la descripción del caso Gödel-Schmidt-John}

Ludwig (2007) y Deutsch (2009) han objetado que el cuestionario usado por Machery et al. (2004) era equívoco. La principal ambigüedad que destacan concierne a la diferencia entre referencia del hablante y referencia semántica, tematizada en Kripke (1977). No prejuzgamos ninguna cuestión si hacemos esa distinción de forma muy simplificada indicando que cuando $\mathrm{H}$ usa el término referencial $t$ en un acto de habla, la referencia del hablante es la entidad a la que $\mathrm{H}$ intenta referirse al usar $t$, mientras que la referencia semántica es la entidad a la que de hecho se refiere al usar $t$; si todo funciona bien, una y otra referencia coinciden; pero no siempre es así. El debate descriptivismo/anti-descriptivismo versa sobre la referencia semántica. Sin embargo, la pregunta que se hizo a los encuestados en los experimentos de Machery et al. (2004) bien podía ser interpretada como una cuestión sobre la referencia del hablante (cf. Ludwig 2007; Deutsch 2009; Sytsma y Livengood 2011; Ichikawa et al. 2012). Quienes tomaran la pregunta en ese sentido no están manifestando ninguna

4 Es decir, si las encuestas se hubieran efectuado impecablemente y fuera correcta la interpretación de las mismas por parte de Machery et al. En las próximas secciones justificaré que hay indicios de peso contra cada uno de esos dos supuestos. 
intuición semántica descriptivista respecto a la referencia semántica, que es lo relevante, si optan por la respuesta $A$.

En Machery et al. (2015) se presentan los resultados de otra serie de estudios estadísticos muy similares a los anteriores. Una de las novedades tiene como finalidad sortear la dificultad antedicha, sobre la ambigüedad entre referencia del hablante y referencia semántica. Las respuestas A y B son las mismas, pero ahora la pregunta ante la cual se pide a los sujetos que escojan A o B es otra: «independientemente de de quién podría intentar hablar [regardless of whom he might intend to be talking about], ¿de quién habla realmente John cuando usa el nombre 'Gödel'?».' Éstos son los resultados: En el grupo de estadounidenses, el 40,5\% respondió A, el 59,5\% respondió B. En el grupo de asiáticos, el 61,2\% respondió $\mathrm{A}$, el $38,8 \%$ respondió $\mathrm{B}$.

Desde mi punto de vista, ese cambio no reduce suficientemente la ambigüedad. Veremos en ésta y en las próximas tres secciones diversos elementos que contribuyen a la falta de claridad del diseño experimental, también en su nueva versión.

Machery et al. pretenden evaluar ituiciones sobre el concepto de referencia. Sin embargo, no preguntan explícita y directamente a quién se refiere John, o a qué nombra (o a qué refiere) el término 'Gödel' cuando lo usa John. Preguntan mediante esa otra noción, de quién habla John, incluso cuando - una vez alertados de que el cuestionario puede ser equívoco- intentan evitar que la cuestión se tome como una cuestión sobre la referencia del hablante. Pese a ello, en el texto describen su test como si éste preguntara explícitamente por la referencia (Machery et al. 2015, p. 69). No hubiera sido muy complicado presentar a los encuestados una pregunta que redujera todavía más el riesgo de que los sujetos entendieran que se les interroga sobre la referencia del hablante, algo como «independientemente de a quién podría intentar referirse John, ¿a quién se refiere realmente John cuando usa el nombre 'Gödel'?», o como «independientemente de a quién podría intentar referirse John, ¿qué persona es nombrada por John cuando usa el nombre 'Gödel'?».

Hay un problema bastante más grave. El caso Gödel-Schmidt-John, tal y como se presentó literalmente a los encuestados, es — al menos a primera vista - inconsistente. En la exposición del caso se indica primero que John ha aprendido [learned] que Gödel descubrió la incompletud de la aritmética y luego se indica que el descubridor no fue Gödel. Los diccionarios señalan que aprender implica conocer (lo aprendido), y 'conocer' es un verbo factivo. Así pues, 'aprender' es también factivo. (Análogamente, respecto a 'to learn' y 'to know'.) Aseverar que John aprende que Gödel descubrió la incompletud de la aritmética y que el descubridor no fue Gödel es incurrir en una contradicción. ${ }^{6}$

Por consiguiente, para que los encuestados entendieran de forma coherente el caso Gödel-Schmidt-John tuvieron que hacer una interpretación caritativa no literal del relato. Pero entonces, ¿qué garantía tenemos de que cuando se les pregunta por de quién

5 Si se prefieriera una traducción menos cacofónica, aunque quizá no tan literal, léase «independientemente de sobre quién podría intentar hablar, ¿sobre quién habla realmente John...?».

6 A mi juicio, también es contradictorio decir que Gödel no descubrió la incompletud de la aritmética pero ha sido conocido [has been known] como el hombre que descubrió la incompletud de la aritmética. Algunas personas considerarán, sin embargo, que aunque 'conocer' sea factivo, la locución ' $x$ es conocido como el F' no implica literalmente que $x$ es el F. No necesito entrar en la discusión de esa cuestión. El problema relativo al carácter factivo de 'aprender' ya es suficiente para formular la objeción principal que resalto en esta sección. 
habla John lo interpreten como Machery et al. requieren que lo interpreten: como una pregunta sobre a qué nombra (o a qué refiere) literalmente el término 'Gödel' cuando lo usa John?

Es conveniente perfilar con mayor detalle y detenimiento en qué consiste esta crítica, así como comentar algunos textos que - directa o indirectamente- inciden sobre la cuestión. (Un/a anónimo/a evaluador/a de Theoria me ha hecho ver la conveniencia de ampliar el tratamiento de esta problemática.) Así, por ejemplo, Ichikawa et al. (2012, nota 2) mencionan este inconveniente (la inconsistencia de la descripción del caso Gödel-Schmidt-John), pero apenas le conceden importancia. Por otro lado, tanto Kallestrup (2016) como Beebee y Undercoffer (2016) hacen referencia al problema y presentan propuestas específicas. Por su parte, Hazlett (2011) - sin abordar el debate concreto entre el anti-descriptivismo de Kripke y la «filosofía experimental» de Machery et al.- niega que 'saber' y 'aprender' sean verbos factivos. Haré algunas consideraciones sobre esos tres últimos textos.

He indicado que 'aprender', así como también el verbo inglés 'to learn', es un verbo factivo. Enseguida indicaré por qué me parece que es así y exploraré alguna posible réplica a esa tesis. No obstante, conviene señalar que la objeción a Machery et al. no depende estrictamente de que 'aprender' sea un verbo factivo. Aunque la objeción tendría poca fuerza si resultara muy patente que 'aprender' no es factivo. El mero hecho de que sea sensato suponer que una proporción significativa de los sujetos podían presuponer la factividad de 'aprender' constituye un problema para Machery et al. Sólo si resulta muy claro que 'aprender' no es factivo podríamos legítimamente suponer que ésa es también la opinión muy mayoritaria de los sujetos encuestados; en cuyo caso, no habría aquí indicio de que al menos una proporción significativa de los sujetos tuvieron que interpretar de forma no literal el caso Gödel-Schmidt-John. Pero dicho supuesto, el supuesto de que 'aprender' es claramente no factivo, resulta - cuando menos- muy controvertido.

En cualquier caso, examinemos la posible verdad o falsedad de la tesis principal, la tesis de que 'aprender' es factivo. ¿Qué tipo de justificación tenemos para aceptar la factividad de 'aprender'? Es el mismo tipo de justificación que la que tenemos para aceptar que 'esconder' es un verbo transitivo, o que estar soltero es incompatible con estar casado. Aunque en todos esos casos - y otros similares - los indicios que apoyan la correspondiente tesis puedan ser falibles, son indicios con suficiente peso. Podemos invocar nuestras propias intuiciones, así como también la consulta a uno o varios diccionarios. En el caso de 'aprender' seguramente el convencimiento de su factividad pasa por su vínculo con 'conocer': nuestras intuiciones y los diccionarios establecen que ' $\mathrm{X}$ ha aprendido que $\mathrm{P}$ ' implica ' $\mathrm{X}$ ha adquirido el conocimiento de que P'. A su vez, nuestras intuiciones y los diccionarios establecen que 'conocer' es factivo.

Naturalmente, todo eso puede ser objeto de controversia. Algunas personas alegarán que sus intuiciones sobre 'aprender', 'conocer', 'esconder', 'soltero' o 'casado' no coinciden con lo que estoy describiendo. También puede haber diccionarios que - en lo que propongan sobre el significado de esas expresiones- entren en contradicción con los diccionarios a los que me he referido. Consideremos la presunta factividad de 'conocer'. Hazlett (2011) la rechaza. Se basa, principalmente, en la existencia de oraciones que, en opinión de Hazlett, la gente corriente no considera inaceptables, impropias, necesariamente falsas, etc. (cf. Hazlett 2011, pp. 500-501). Entre los ejemplos, menciona una frase de la película Titanic: «Todo lo que sabe es erróneo» [Everything he knows is wrong]. 
Enumero a continuación tres inconvenientes, seguidos de una reflexión adicional; no entraré a fondo en el desarrollo de esos puntos, por razones de espacio y porque - como reitero en la reflexión adicional- la objeción contra Machery et al. no requiere que 'conocer' y 'aprender' sean factivos.

a) Hazlett no justifica su opinión de que la gente corriente no considera inaceptables, impropias, necesariamente falsas, etc. las frases que presenta.

b) Supongamos, de todos, modos que esa opinión fuera correcta. Supongamos, incluso, que la gente corriente acertara al tener esos juicios. Nada de eso implica que 'conocer' no sea factivo. La factividad concierne a la verdad literal de ciertas oraciones; no a si las oraciones son aceptables, apropiadas, etc. Que sean aceptables o apropiadas ciertas oraciones literalmente falsas puede ser explicado recurriendo, por ejemplo, a la teoría de las implicaturas conversacionales de Grice (1975). Hazlett, por el contrario, invoca a Grice para explicar por qué 'conocer' parece muy claramente factivo.

c) Supongamos, incluso, que las oraciones citadas por Hazlett, como «Todo lo que sabe es erróneo [wrong] », fueran literalmente verdaderas. He aquí una objeción ad hominem, que cuestiona la legitimidad dialéctica de la posición de Hazlett: Hazlett no justifica por qué, según su criterio, eso implicaría que 'saber' no es factivo. Yo sí creo, y supongo que conmigo muchos lectores, que la supuesta verdad de «Todo lo que $\mathrm{X}$ sabe es erróneo [wrong]» (junto con el supuesto de que $\mathrm{X}$ sabe alguna cosa) sería incompatible con la factividad de 'saber'. Pero si me pregunto por la justificación de esa creencia, constato que depende de mis intuiciones sobre el significado de 'erróneo' y quizá de la consulta a algún diccionario. No son indicios relevantemente más firmes que los indicios de que 'saber' es factivo (¿por qué habrían de serlo?). Hazlett cuestiona los indicios favorables a la tesis que rechaza (la factividad de 'saber') basándose en una tesis, para la cual no aporta indicio alguno, que no sería menos cuestionable que esa tesis principal por él rechazada.

Vayamos con la reflexión adicional. La objeción que he planteado contra Machery et al. mantiene parte de su fuerza incluso si 'saber' y 'aprender' - como pretende Hazlett - no son factivos. Ciertamente, Machery et al. podrían pretender que muchos sujetos encuestados asumían, acertadamente o erróneamente, que 'aprender' no es factivo; esos sujetos podrían interpretar todo el test literalmente, conforme a lo que ellos creen que significan las palabras usadas en él. Pero esa pretensión no anula el fondo de esta crítica. Bajo la hipótesis de que 'aprender' es factivo: si un sujeto encuestado asumía que 'aprender' no es factivo, ¿qué razones tenemos para suponer que consideraba equivalentes el concepto $h a-$ $b l a r-d e-x$ y el concepto referirse-a-x? ¿qué razones tenemos, en general, para suponer que interpretaba literalmente todas las restantes partes del test (que lo interpretaba literalmente, conforme a lo que nosotros creemos que significan las palabras usadas en él; pues eso es lo relevante para que nosotros evaluemos si los resultados confieren algún apoyo a las tesis M1 y M2)? Bajo la hipótesis de que 'aprender' no es factivo: esta hipótesis dista mucho de ser obviamente verdadera; los sujetos encuestados contrarios a la hipótesis interpretaron el test de forma (según su criterio) no literal; ningún dato hace pensar que constituían un grupo tan minoritario que su presencia no desvirtuaba los resultados de las encuestas. 
También Kallestrup (2016, pp. 50-51) menciona la aparente factividad de 'aprender' y de 'conocer'. Con vistas a minimizar la gravedad del problema que eso supone para Machery et al., hace una propuesta en la cual invoca una diferencia (en los modos posibles de interpretar el caso Gödel-Schmidt-John) en perspectiva doxástica: la perspectiva del narrador no coincide con la perspectiva de John. ${ }^{7}$ Aunque Gödel no descubriera la incompletud de la aritmética, atribuir a John el conocimiento de que Gödel descubrió la incompletud de la aritmética es consistente — según Kallestrup - con que 'conocer' sea factivo, porque esa atribución se hace bajo ciertas suposiciones: las suposiciones de John, lo que John cree que es verdad (cf. Kallestrup 2016, p. 51). Me parece una solución errónea. Esa estrategia implicaría que las atribuciones de conocimiento se relativizan a las suposiciones o creencias de las personas. Así, cualquier creencia C constituiría conocimiento; basta escoger apropiadamente las creencias relativamente a las cuales la atribución de conocimiento es correcta; incluir $\mathrm{C}$ entre tales creencias lo garantizaría.

La contrarréplica que Beebee y Undercoffer (2016, sección 6) ofrecen al problema de la factividad de 'aprender' resulta sorprendente. Contestan a un problema diferente, o bien no han entendido el problema en toda su generalidad. Los encuestados reciben dos presuntas informaciones, aparentemente inconsistentes: «John ha aprendido $\mathrm{P} \gg \mathrm{y}$ «No $\mathrm{P}$ ». Aunque Beebee y Undercoffer mencionan que los filósofos suelen asumir que 'aprender' es factivo, creen que la dificultad deriva del orden en que se presentan las dos presuntas informaciones: sólo se generaría una inconsistencia porque el supuesto dato «John ha aprendido $\mathrm{P} \gg$ es presentado en primer lugar. Para evitarlo, han elaborado estudios estadísticos en los cuales se invierte ese orden, pero a los encuestados todavía se les indica «John ha aprendido $\mathrm{P} \gg$, después de habérseles indicado «No $\mathrm{P} \gg$. No dudo de que el orden en que se presentan los supuestos datos puede afectar a nuestra aceptación de los mismos. Sin embargo, es muy difícil convencerse de que ese mero cambio de orden subsana el problema.

\section{Descriptivismo débil versus descriptivismo fuerte}

En esta sección mostraré que, independientemente de la equivocidad y la inconsistencia señaladas en la sección anterior, hay ulteriores problemas de ambigüedad, que — por lo que yo sé- no se han destacado en la literatura. Concretamente, argumentaré que la respuesta $\mathrm{A}$ del test puede interpretarse como una respuesta no descriptivista. Kallestrup (2016) ha dado a entender una tesis similar; si bien en otro pasaje parece comprometerse con la negación de la tesis; cf. nuestra próxima sección. Sin embargo, la explicación que propongo a favor de dicha tesis es muy diferente a la de Kallestrup.

Atendamos a las diferentes implicaciones de las versiones débil y fuerte del descriptivismo. Kripke acepta que existen algunos nombres descriptivos, respecto a los cuales sería correcto el descriptivismo débil; niega que los nombres sean mayoritariamente de ese tipo. Supongamos que estipulamos llamar 'Julius' a la persona, quienquiera que fuera, que inventó la cremallera (recurro al célebre ejemplo de G. Evans). Mientras no transcurra más historia, 'Julius' es un nombre descriptivo. Asociamos con 'Julius' la descripción 'el inventor

7 La diferencia entre ambas perspectivas procede de Sytsma y Livengood (2011). En nuestra sección 5 volveremos sobre ella. 
de la cremallera', y respecto a 'Julius' son verdaderas — dados los hechos en el mundo reallas dos tesis análogas a las tesis (i) y (ii) que serían falsas respecto a 'Gödel' si los hechos fueran conforme a lo narrado en el caso Gödel-Schmidt:

(i*) Para todo $x$, si $x$ es el inventor de la cremallera, entonces 'Julius' nombra (refiere) a $x$

(ii**) Para todo $x$, si 'Julius' nombra (refiere) a $x$, entonces $x$ es el inventor de la cremallera

Dada esa estipulación, y suponiendo que existe un único inventor de la cremallera, nos resulta ininteligible (si no estamos en una fase diferente, más avanzada, de la posible historia de los usos del nombre) considerar la hipótesis de que Julius no inventó - en el mundo real - la cremallera. Pero es perfectamente inteligible considerar situaciones contrafácticas en que Julius no es el invenor de la cremallera: podemos preguntarnos qué habría pasado si Julius hubiera muerto a los 18 meses de edad, y concluir que no habría llegado a inventar nada (cf. Pérez Otero 2006, sección 3.2; Pérez Otero 2013, pp. 259-264). Si el descriptivismo fuerte es correcto, entonces (aplicado a este caso) también esa segunda consideración hipotética resulta dudosamente inteligible; la descripción 'el inventor de la cremallera' no sólo fijaría la referencia de 'Julius', sino que ambas expresiones serían sinónimas; 'Julius' sería aproximadamente equivalente a una abreviatura de 'el inventor de la cremallera'.

Generalicemos. Supongamos que 'el F' es (conforme al descriptivismo) la descripción prioritariamente asociada con el nombre propio NP. Supuesto el descriptivismo débil, y suponiendo también que existe un único F, es ininteligible la hipótesis 'NP no es el F' (considerada como una hipótesis sobre el mundo real y asumiendo también que no estamos en una fase más avanzada de la posible historia de los usos del nombre NP), pero es inteligible suponer que en otra situación contrafáctica NP no sea el F. Supuesto el descriptivismo fuerte, ambas hipótesis son ininteligibles.

Consideremos ahora cómo puede/debe interpretarse el caso Gödel-Schmidt original de Kripke y cómo puede/debe interpretarse el caso Gödel-Schmidt-John.

En el experimento mental ideado por Kripke no hay una separación entre un John, de un lado, y unos sujetos interrogados sobre usos del nombre por parte de John, del otro. Ambos papeles los desempeñamos cualquiera de nosotros. Antes de plantear el caso GödelSchmidt, Kripke ha justificado con otros ejemplos, como el nombre 'Feynman', que en ocasiones usamos un nombre propio y no creemos poder asociar con él una descripción definida identificadora. Para otros nombres propios asociamos - correcta o incorrectamente - con su referente bastante material descriptivo (diversas descripciones definidas) presuntamente identificador. Hay muchos casos situados entre ambos extremos: nombres propios sobre cuyo referente creemos saber que cumple cierta descripción definida que podemos exhibir, pero para el cual no sabríamos invocar descripciones identificadoras adicionales. Supongamos entonces - nos propone Kripke - que 'Gödel' sea un nombre de ese tercer grupo y 'el descubridor de la incompletud de la aritmética' la correspondiente descripción asociada. Como he sugerido antes, la forma metodológicamente más apropiada de enfocar el caso por parte de cada lector/sujeto, $S$, sería que $S$ aceptara la invitación implícita de Kripke y manejara su propio ejemplo de un nombre NP para el cual sólo sabría asociar cierta descripción definida. En ese sentido somos como John: creemos haber aprendido que Gödel (o el referente del nombre que escojamos como ejemplo) descubrió la incompletud de la aritmética y eso es todo lo que creemos saber de él. 
Debemos tener en cuenta esa situación dialéctica al reflexionar sobre cada una de las dos maneras en que podría interpretarse el caso Gödel-Schmidt:

(R) Supongamos que (hay un único descubridor de la incompletud de la aritmética pero), contra lo que habiamos creído, Gödel no es el descubridor de la incompletud de la aritmética. Si es así, no necesitamos preguntarnos cuál creemos que es la referencia de 'Gödel'. Análogamente a lo indicado sobre 'Julius', el mero hecho de que entendamos sin dificultad esa suposición está en conflicto incluso con el descriptivismo débil. Más exactamente, quien entienda sin dificultad esa suposición muestra con ello que su manejo de 'Gödel' es inconsistente con el descriptivismo débil.

(C) Supongamos que Gödel es el descubridor de la incompletud de la aritmética; pero consideremos una situación contrafáctica en que Gödel, contra lo que se cree en dich a situación, no hubiera sido el descubridor de la incompletud de la aritmética. Conforme a esta interpretación contrafáctica del caso, la mera comprensión de esa suposición no contradice el descriptivismo débil; sólo contradice el descriptivismo fuerte.

Kripke no extrae resultados del caso Gödel-Schmidt basándose sólo en nuestra mera comprensión del caso. Apela a cuál nos parece que sería el referente de 'Gödel'. En efecto, lo más sensato es suponer que no exhibe el caso para desmontar el descriptivismo fuerte, sino para desmontar el descriptivismo débil. Así pues, la interpretación contrafáctica, (C), sería la adecuada. ${ }^{8}$ Aunque conviene no olvidar esa otra fuerza dialéctica presente en el caso GödelSchmidt: si lo que está en cuestión es el descriptivismo fuerte, meramente entender sin dificultad el caso - incluso bajo su interpretación contrafáctica - ya revela una posición contraria a dicho descriptivismo.

Vayamos con el caso Gödel-Schmidt-John. John estaría, respecto a las expresiones 'Gödel' y 'el descubridor de la incompletud de la aritmética', en una situación como la nuestra recién descrita. No tenemos datos sobre la situación de los encuestados. Bajo la suposición de que un sujeto encuestado, $S$, estuviera también en esa situación (que sólo asociara con 'Gödel' la descripción 'el descubridor de la incompletud de la aritmética') tenemos lo siguiente. Que $S$ entienda el caso narrado (tanto si lo entiende al modo (R), como una hipótesis acerca del mundo real, como si lo entiende conforme a (C), como una hipótesis contrafáctica) revelaría que su posición es contraria al descriptivismo fuerte. Machery et al. (2015) declaran que su tema es el descriptivismo débil (cf. su nota 5). Si S entiende el caso narrado al modo (R), ello mostraría que su posición es contraria al descriptivismo débil.

La crítica que acabo de mencionar (si el caso Gödel-Schmidt-John se entiende conforme a $(\mathrm{R})$, entonces la mera comprensión del caso — por parte de un sujeto, S, que sólo asociara con 'Gödel' la descripción 'el descubridor de la incompletud de la aritmética - es contraria incluso al descriptivismo débil) también es sugerida por Martí (2009, p. 46). Pero Martí se muestra reticente ante este tipo de crítica. Desde su punto de vista, si los encuestados sabian que el caso narrado era un caso contrafáctico, entonces las posiciones de esos encuestados son consistentes con el descriptivismo débil. En otras palabras, si los encuestados sabian que la interpretación apropiada del caso es $(C)$, entonces la mera comprensión del caso es consistente con el descriptivismo débil. Coincido con ella respecto a ese condicional; pero no soy tan reticente, precisamente porque no tenemos datos como para garantizar

8 Kallestrup (2016) sin embargo, considera que tanto el caso Gödel-Schmidt como el caso GödelSchmidt-John deben interpretarse en un sentido cercano a $(\mathrm{R})$; cf. nuestra próxima sección. 
que se cumple el antecedente del condicional; la interpretación (R) no parece descartable de antemano.

De todos modos, exploremos la opción preferible para Machery et al.: supongamos que el sujeto encuestado, $S$, interpreta el caso narrado en sentido contrafáctico, análogo a (C). ¿Por qué es A una respuesta descriptivista? La (principal descripción definida contenida en la) respuesta $\mathrm{A}$ tiene dos interpretaciones naturales, $\mathrm{AR}$ y $\mathrm{AC}$ :

\section{A: la persona que realmente descubrió la incompletud de la aritmética}

AR: la persona que en el mundo real descubrió la incompletud de la aritmética (es decir, Gödel)

AC: la persona que en esa situación contrafáctica descubrió la incompletud de la aritmética (es decir, Schmidt)

Si un encuestado interpreta A en el sentido AR, su opción por esa respuesta es consistente con la negación del descriptivismo débil, contrariamente a lo que pretenden Machery et al. Pero la lectura AR de A no es forzada, resulta perfectamente legítima. Tenemos, pues, otra muestra de cómo la equivocidad del diseño experimental lastra severamente las conclusiones propuestas.

Concedamos, sin embargo, que en el marco general del test, interpretar A en el sentido AC parece la opción más caritativa que los sujetos encuestados pudieran tomar. Es así porque A se les presenta como alternativa a B:

\section{B: la persona que se apropió del manuscrito y se atribuyó el mérito}

Puesto que estamos considerando la interpretación contrafáctica del caso, sería impropio tomar B en el sentido BR, dado que en el mundo real nadie se apropia de un manuscrito ajeno sobre la incompletud de la aritmética:

BR: la persona que en el mundo real se apropió del manuscrito y se atribuyó el mérito (nadie)

BC: la persona que en esa situación contrafáctica se apropió del manuscrito y se atribuyó el mérito (es decir, Gödel)

Los sujetos encuestados seguramente interpretarían B en el sentido BC. Ésa es la razón para pensar que, análogamente, probablemente también interpretarían A en el sentido AC (no en el sentido AR, compatible con la negación del descriptivismo débil). La posición de Machery et al. dependería, entonces, de suponer que los sujetos no interpretan la respuesta A por sí misma, sino en conexión con lo que suponen que dicha respuesta significa basándose también en lo que pueda significar la respuesta $B$.

Con todo, desde mi punto de vista, la opción de leer A al modo AR no resulta tan remota como sería necesario para reducir suficientemente la equivocidad del test. Esta consideración - A podría interpretarse coherentemente con la negación del descriptivismo débil - se suma al problema de inconsistencia ya señalado y a los otros problemas sobre ambigüedad (incluyendo el que presento en la sección 6).

\section{Otras posibles ambigüedades}

Tras acabar una primera versión de este artículo he tenido conocimiento de que un texto reciente, Kallestrup (2016), aparentemente también sostendría —en su tramo final— la 
tesis de que A no es una respuesta necesariamente descriptivista. Pero Kallestrup ofrece en favor de esa tesis una razón muy diferente de la mía. Un/a evaluador/a de dicha versión previa de mi texto ha sugerido que algunas de mis críticas a Machery et al. podrían ser semejantes a críticas formuladas por otros autores, por lo cual sería conveniente especificar en qué sentido es novedosa mi posición. En particular, menciona Sytsma y Livengood (2011) y Kallestrup (2016), los cuales — según ese/a evaluador/a - contendrían alusiones a problemas de ambigüedad muy cercanos al problema concreto que he destacado en la sección anterior. ${ }^{9}$

Dedicaré esta sección a mostrar que la ambigüedad crucial de los tests de Machery et al. que presento en esa sección 4 difiere de las otras ambigüedades postuladas en esos dos textos. También difiere, concretamente, de la ambigüedad en la cual se basaría Kallestrup para, en apariencia, sostener que la respuesta A podría interpretarse de forma no descriptivista. Para facilitar la comparación, primero resumiré escuetamente mis reflexiones principales de la sección $4 .^{10}$

He distinguido el caso Gödel-Schmidt del caso Gödel-Schmidt-John pues nadie —ni Machery et al. ni ninguno de los textos comentados en esta sección- ha ofrecido razón alguna para suponer que los diversos modos en que puede o debe interpretarse el caso Gödel-Schmidt hayan de coincidir exactamente con los diversos modos en que puede o debe interpretarse el caso Gödel-Schmidt-John. Luego he discutido qué opciones interpretativas existen respecto a cada uno de los dos casos, por separado. Respecto al caso GödelSchmidt, he destacado la ambigüedad entre la lectura (R) y la lectura (C), defendiendo que parece más apropiado entender que Kripke proponía el caso en el sentido contrafáctico, (C). Respecto al caso Gödel-Schmidt-John, estarían abiertas ambas opciones, (R) y (C). La lectura (R) resultaría muy problemática para Machery et al. Asumiendo la lectura más caritativa para con Machery et al., la lectura (C), he señalado una nueva ambigüedad: entre las interpretaciones AR y AC. Es esta segunda ambigüedad — no la ambigüedad (R) versus (C) - la que importa especialmente: he señalado que si los encuestados entienden la respuesta $A$ conforme al sentido $A R$, entonces la respuesta $A$ no es una respuesta descriptivista.

9 Desde un punto de vista muy general, algunos aspectos de mis objeciones al diseño experimental de Machery et al. son cercanos al enfoque de Lam (2010). Sin embargo, Lam basa sus reflexiones en estudios estadísticos que contienen, además de algunos de los errores que atribuyo a Machery et al., otro error quizá más grave. Lam construye un caso en el cual los nombres 'Shakespeare' y 'Spencer' desempeñan un papel análogo al de los nombres 'Gödel' y 'Schmidt' en el caso Gödel-Schmidt-John. Pero en una de las dos preguntas que se les pasó a los encuestados se les preguntaba si al usar el nombre 'Shakespeare' se hablaba sobre Shakespeare (opción A) o sobre Spencer (opción B). Y afirma que «las respuestas «A $\mathrm{A}$ son acordes [consonant] con la teoría histórico-causal de los nombres, las respuestas «B» son acordes con la teoría descriptivista de los nombres.» (p. 322), sin apercibirse de un hecho que desvirtúa ese planteamiento: esa respuesta A es también acorde con la teoría descriptivista. Un descriptivista no tiene razones para rechazar tesis desentrecomilladoras sencillas, del tipo: si 'Shakespeare' es un nombre propio y tiene denotación, la denotación de 'Shakespeare' es Shakespeare. (Cf., asimismo, Beebee y Undercoffer 2016.)

${ }^{10}$ Mi objetivo aquí no es emprender una evaluación crítica de las diferentes tesis y opiniones expresadas en esos dos artículos. Sólo me propongo clarificar las diferencias entre mis tesis y aquellas de sus tesis que pudieran aparecer como similares a las mías. 
Sytsma y Livengood (2011) destacan dos posibles ambigüedades presentes en las pruebas de Machery et al. Una es la ambigüedad — señalada inicialmente por Ludwig (2007) - entre referencia del hablante y referencia semántica, de la que también me he hecho eco en la sección 3. La otra sería una ambigüedad de perspectiva doxástica, o epistémica, consistente en lo siguiente. A los sujetos encuestados se les narra una situación que contiene a un determinado individuo, John. Los hechos presentados como verdaderos por el narrador no coinciden exactamente con los hechos que John considera verdaderos. Así, la perspectiva doxástica del narrador difiere de la perspectiva doxástica de John. Según Sytsma y Livengood, cuando los encuestados son interrogados sobre de quién habla John al usar el nombre 'Gödel' existiría esta ambigüedad: podrían interpretar que se les pregunta conforme a la perspectiva del narrador, o bien conforme a la perspectiva de John; si se interpreta según esa segunda perspectiva, la pregunta «¿de quién habla John al usar el nombre 'Gödel'? » sería equivalente a «¿de quién cree John que habla al usar el nombre 'Gödel'?».

Esa posible ambigüedad de perspectiva doxástica no es equivalente a la ambigüedad entre las lecturas $(\mathrm{R})$ y $(\mathrm{C})$ que he resaltado. Tampoco es equivalente a la ambigüedad -más importante- entre las lecturas AR y AC, que surge sólo si asumimos la lectura (C) del caso Gödel-Schmidt-John. En esas diferentes interpretaciones que he postulado en la sección 4 he asumido que los encuestados adoptaban la perspectiva del narrador. No he contemplado la hipótesis de que contestaran como si se les preguntara por la perspectiva de John.

Kallestrup (2016, sección 4) invoca esa distinción entre perspectiva del narrador y perspectiva de John; aunque modifica la caracterización de la distinción que proponían Sytsma y Livengood. En un pasaje, Kallestrup propone que si un sujeto encuestado adopta la perspectiva de John, entonces su respuesta A es compatible con el descriptivismo pero también con el anti-descriptivismo (p. 52). Coincidimos, pues, en lo siguiente: Kallestrup señala una ambigüedad (perspectiva del narrador versus perspectiva de John) y sostendría (aparentemente) que conforme a una de las dos lecturas (la perspectiva de John) A no es necesariamente una respuesta descriptivista. Yo he señalado otra ambigüedad (lectura AR versus lectura AC) y sostengo que conforme a una de las dos lecturas (la lectura AR) A no es necesariamente una respuesta descriptivista. Reitero que la ambigüedad de perspectivas doxásticas - apuntada por Sytsma y Livengood, y retomada por Kallestrup — no desempeña ningún papel en mis propuestas.

Vengo resaltando que sólo en apariencia acepta Kallestrup la tesis de que A no es una respuesta necesariamente descriptivista debido a sus comentarios en la nota 25, que parecen contradecir esa posición. Kallestrup escribe: «whenever a participant reports a descriptivist intuition, she must be expressing a descriptivist intuition, whatever perspective she adopts» Kallestrup (2016, nota 25). Creo que sus opiniones sobre la significación de la distinción entre perspectiva del narrador y perspectiva de John resultan algo confusas, y en parte erróneas (cf. también mis comentarios de la sección previa, sobre su propuesta de relativizar las atribuciones de conocimiento a las perspectivas doxásticas). Pero —como ya he indicado- no es mi tarea aquí evaluar críticamente sus tesis, sino sólo especificar lo novedoso de mis propuestas respecto a las suyas.

En una sección previa (sección 3) Kallestrup establece otra diferencia o ambigüedad, que - a grandes rasgos - se correspondería con la ambigüedad entre las lecturas (R) y (C). Resalta un contraste entre la interpretación que llama counteractual de los casos y la interpretación contrafáctica [counterfactual]. Esta última sería la lectura (C), mientras que la 
interpretación counteractual puede asimilarse a la lectura (R). ${ }^{11}$ Según Kallestrup, el caso Gödel-Schmidt y el caso Gödel-Schmidt-John deben interpretarse en ese sentido counteractual; también en eso discrepo de él. Sea como fuere, no hay en su texto ningún atisbo de la ambigüedad AR versus AC, en la cual baso mi afirmación de que la respuesta A no necesariamente es descriptivissta; y dicha ambigüedad sólo surge en el seno de la lectura cotrafáctica. Por el contrario, él basa esa afirmación en el contraste entre perspectivas doxásticas; contraste presentado en el seno de la lectura counteractual.

\section{Las encuestas en lengua china}

En Machery et al. (2015) se describe una novedad adicional. A otro grupo de universitarios asiáticos encuestados se les presentó toda la prueba — relativa a la pregunta «independientemente de de quién podría intentar hablar, ¿de quién habla realmente John cuando usa el nombre 'Gödel'? » - en lengua china. He aquí los resultados: el 81,5\% respondió A, el 18,5\% respondió B. Recordemos los porcentajes de respuestas de los universitarios asiáticos encuestados en inglés: el 61,2\% respondió A, el 38,8\% respondió B. Las características de ambos grupos eran muy similares; se trataba de estudiantes jóvenes de la Universidad de Hong Kong y de la Universidad de Ciencia y Tecnología de Hong Kong. Machery et al. (2015) no destacan ninguna otra diferencia potencialmente relevante (aunque señalan que el porcentaje de varones era de $71 \%$ en el grupo encuestado en inglés y de $46 \%$ en el otro grupo; en el grupo estadounidense era $24,1 \%$ ). Cabe suponer que los sujetos de ambos grupos eran hablantes del inglés pero tenían el chino como lengua materna.

Machery et al. (2015) minimizan la importancia de esa considerable discrepancia, y no aventuran ninguna explicación (cf. su nota 18). Pero creo que el dato llama a la reflexión. Como es sabido, la validez de los resultados experimentales en cualquier disciplina teórica depende de su carácter replicable. Ello conduce a pensar lo siguiente (siempre bajo la hipótesis de que los resultados presentados en Machery et al. 2015 tengan validez): si el mismo grupo de sujetos que fueron encuestados en chino hubieran sido encuestados en inglés, el resultado se habría aproximado al de sus colegas asiáticos encuestados en inglés (respuesta A: $61,2 \%$, respuesta B el 38,8\%). Así pues, aparentemente las intuiciones supuestamente descriptivistas de esos sujetos - las intuiciones que Machery et al. intentan medir- dependen de la lengua en que se realiza la prueba. ¿Qué tipo de explicación de esa variabilidad es aceptable? ¿Qué hipótesis congenian mejor con esa posible explicación?

Desde mi punto de vista, esos hechos congenian mal con la hipótesis de que las preguntas eran claras e inequívocas y transmitían con eficacia contenidos semánticos literales, suficientemente independiente de especifidades lingüísticas de las palabras concretas utilizadas para expresarlos. Todo ello redunda en las dudas ya planteadas en las secciones 3 y 4 : hay ra-

${ }^{11}$ Elucida la idea de la interpretción counteractual indicando que conciernen a un sentido epistémico de la modalidad, a cómo las cosas podrían haber resultado ser en el mundo real (no en otro mundo posible), para lo cual recurre a ideas de Chalmers y del bi-dimensionalismo (aunque también Kripke 1972/1980 hace alusiones a ese sentido epistémico de la modalidad; por ejemplo cuando menciona que quien crea que las verdades matemáticas son necesarias puede, sin embargo, decir que cierta proposición matemática cuyo valor de verdad se desconoce podría resultar ser verdadera y podría resultar ser falsa). 
zones de peso para considerar que los cuestionarios utilizados, también en 2015, han sido excesivamente ambiguos.

\section{Sobre el papel de las intuiciones en la filosofia: Marti (2009)}

En esta sección y en la siguiente comentaré y defenderé parcialmente ciertos aspectos de la posición de Machery et al., relativos a su evaluación del papel de las intuiciones en los casos Gödel-Schmidt y Gödel-Schmidt-John. Otros aspectos de esa posición se critican en la sección 9.

Recordemos las dos tesis - relacionadas entre sí- que Machery et al. intentan justificar mediante los resultados de sus experimentos: (M1) casos del tipo Gödel-Schmidt no respaldan el anti-descriptivismo; (M2) las supuestas intuiciones anti-descriptivistas puestas de relieve en casos del tipo Gödel-Schmidt no son suficientemente generales. Como es natural, se pretende que la tesis (M1) quedaría bien respaldada por la tesis (M2). He presentado objeciones contra (M2), que serían también objeciones contra (M1).

Una de las críticas importantes dirigidas contra Machery et al. (2004), procedente de Martí (2009), niega que estos experimentos sirvan para apoyar (M1), incluso si permitieran sostener (M2). Según Martí, las encuestas de Machery et al. (2004) no preguntan sobre lo más relevante para evaluar la verdad o falsedad del descriptivismo. Si esas encuestas fueran correctas, sólo mostrarían que los orientales son más proclives al descriptivismo que los estadounidenses. Eso es compatible con la verdad de una teoría anti-descriptivista sobre la referencia de los nombres propios. En lugar de detectar qué teoría explícita sobre la referencia sostienen los sujetos encuestados, habría sido preferible — propone Martí- diseñar algún test para detectar cómo usan los nombres propios esos sujetos, y comprobar qué teoría sobre la referencia es consistente o no con tales usos. Sugiere para ello un test diferente: comprobar si los encuestados reaccionarían con naturalidad o extrañeza ante el caso de un John que, tras ser informado del fraude perpetrado, profiriese oraciones como «Nos hemos enterado de que Gödel era un ladrón y un mentiroso». Sólo si los encuestados reaccionaran con extrañeza ante tales oraciones estarían usando 'Gödel' de forma consistente con el descriptivismo.

Hay aquí varios elementos en juego. A mi juicio, las opiniones sinceras de los sujetos sobre cómo deberían aplicarse ciertos conceptos o expresiones lingüísticas (nombres propios, 'referencia', 'significado', o —en otros ámbitos- 'saber', 'bueno', 'causa') en situaciones imaginadas suficientemente simplificadas (experimentos mentales) reflejan bastante bien qué intuiciones tienen sobre las condiciones de aplicación de tales conceptos. Estas intuiciones, a su vez, constituyen un indicio falible pero fiable de la teoría correcta sobre las extensiones de los conceptos en cuestión. Supongamos que los experimentos de Machery et al. no adolecieran de los inconvenientes de inconsistencia y ambigüedad ya mencionados. En tal caso, los resultados serían un indicio importante contra la corrección general de una teoría anti-descriptivista. Incluso en ese caso, serían sólo un indicio falible. ¿Por qué? ¿Por qué, incluso si alguien hubiera conseguido justificar (lo cual no ha sucedido) que aproximadamente el $60 \%$ de los asiáticos tienen intuiciones descriptivistas, la posición epistémicamente más razonable pudiera ser aceptar una teoría anti-descriptivista de la referencia?

Las respuestas englobarían varios factores, incluyendo los apuntados por Martí, así como - desde luego- consideraciones sobre diferencias cualitativas en el peso de las intui- 
ciones: la mejor teorización sobre cómo las teorías filosóficas se apoyan en intuiciones no necesariamente presupondrá que todas las intuiciones valen igual (cf. nuestra sección 9). En lo concerniente a la objeción de Martí, habría una diversidad de correlaciones relevantes: entre las opiniones sinceras explícitas manifestadas por los sujetos y sus intuiciones reales; entre tales intuiciones y el modo en que realmente usan los conceptos en cuestión; entre esas intuiciones y la teoría verdadera sobre las extensiones de los conceptos en cuestión; entre el modo en que usan los conceptos en cuestión y la teoría verdadera sobre sus extensiones. Martí destaca la brecha entre las opiniones sinceras explícitas manifestadas por los sujetos y el modo en que realmente usan los conceptos en cuestión. Parece asumir - con acierto, a mi juicio- que sería comparativamente menos importante la posible brecha entre el uso de los conceptos en cuestión y la teoría verdadera sobre sus extensiones. En ese sentido, para evaluar la tesis (M1) y, en último término, para tomar partido en la disputa descriptivismo/anti-descriptivismo sobre los nombres propios, creo que algún test similar al que ella sugiere tendría mayor relevancia que los diseñados por Machery et al.

No obstante, desde mi punto de vista el test concreto sugerido por Martí podría mejorarse; aunque la modificación lo complicaría un poco. Su test propone presentar a los encuestados la conducta lingüística de un John que usa 'Gödel' consistentemente con una teoría anti-descriptivista, y preguntarles por dichos usos, para detectar su posible extrañeza. Creo que sería preferible presentar a los encuestados dos tipos alternativos de conducta lingüística por parte de John: una conducta consistente con una teoría anti-descriptivista y una conducta consistente con una teoría descriptivista; y preguntar entonces a los encuestados cuál de esas conductas les parece más natural o menos extraña. Seguramente la prueba requeriría especificar cómo se entera John del fraude. Supongamos que estipulamos, por ejemplo, que John lee la noticia del fraude en una revista de historia de la lógica. Sería conveniente entonces presentar a los encuestados estas dos posibles reacciones de John: proferir algo cercano a «Nos hemos enterado de que Gödel era un ladrón y un mentiroso», según proponía Martí; proferir algo cercano a «No entiendo bien esta noticia; ¿cómo pueden decir que Gödel no es realmente el descubridor de la incompletud de la aritmética, si Gödel es [o bien: si llamamos 'Gödel' a] quienquiera que descubrió la incompletud de la aritmática». Me parece preferible ese otro test porque lo considero más neutral. En cualquier caso, mi objetivo aquí no es elaborar con detalle un estudio estadístico alternativo a los de Machery et al., o al sugerido por Martí. Sería ésa una tarea para otro tipo de trabajo.

\section{Sobre el papel de las intuiciones en la filosofia: Deutsch (2009)}

Mis afirmaciones en la sección anterior, así como otras indicaciones en secciones previas, traslucen que estoy atribuyendo a Kripke la invocación - al menos implícita- de nuestras intuiciones cuando evaluamos el caso Gödel-Schmidt. Es indudable que Kripke apela a las intuiciones, incluso explícitamente, en otros pasajes de Naming and Necessity; la intuición (o la impresión intelectual, o la apariencia no perceptiva; como es obvio, las denominaciones pueden diferir) de que ciertos enunciados de identidad no son verdades necesarias desempeña un papel crucial en su argumentación dualista de la tercera conferencia. ${ }^{12}$

${ }^{12}$ Cf. Pérez Otero (2007) para una exposición - y crítica - de dicho argumento, que resalta el papel de tales intuiciones. 
No obstante, Deutsch (2009, p. 445) ha defendido que «nada del argumento famoso de Kripke contra la teoría descriptivista sobre la referencia de los nombres propios depende de asumir algo sobre las intuiciones de la gente». Dejando de lado que me parece inapropiada la primera descripción definida contenida en esa cita (Kripke expone varios argumentos anti-descriptivistas; ninguno destaca como relevantemente más famoso que todos los otros), considero ésa y otras afirmaciones similares de Deutsch desencaminadas. El artículo de Deutsch presenta consideraciones muy valiosas, en especial ideas - que comparto- sobre cómo aplicar una concepción griceana del uso del lenguaje para criticar muchas hipótesis sostenidas en el ámbito de la «filosofía experimental» (pp. 461-464). Aquí solo comentaré, de forma esquemática, su posicionamiento sobre el papel de las intuiciones en la justificación de las teorías filosóficas.

La principal motivación tras esa afirmación de Deutsch parece consistir en el hecho de que una teoría sobre la referencia (o una teoría sobre la causalidad, o sobre el bien) no tiene como tema las intuiciones de nadie. Así, la teoría hace predicciones sobre qué términos refieren a qué referentes, pero «no hace ninguna predicción en absoluto sobre quién intuirá qué» Deutsch (2009, p. 448). Si uno se pregunta cuál es —según Deutsch- la justificación que Kripke ofrecería a alguien que reaccionara ante el caso Gödel-Schmidt diciendo «también en esa situación 'Gödel' nombraría al descubridor de la incompletud de la aritmética», tendrá la impresión de que Deutsch escamotea la respuesta. En algunos pasajes propone que Kripke (así como otros filósofos) no presenta intuiciones en favor de sus tesis, sino argumentos (cf. p. 452). Y afirma que la justificación del juicio de Kripke, sobre el caso Gödel-Schmidt, no depende de si la gente intuye que es verdadero, sino de la calidad del argumento en favor de dicho juicio (cf. p. 465).

Ese contraste entre argumentos e intuiciones es engañoso y erróneo. Para comprobarlo basta - excepto por un elemento diferencial que luego mencionaré- reflexionar sobre cómo las tesis de Deutsch se aplicarían análogamente respecto a una teoría empírica. Una teoría sobre química inorgánica, $\mathrm{T}$, no tiene como tema las observaciones de nadie. $\mathrm{T}$ hace predicciones sobre qué combinaciones de sustancias producirán una combustión, por ejemplo, pero «no hace ninguna predicción en absoluto sobre quién observará qué». Un químico no presenta observaciones en favor de sus tesis, sino argumentos. La justificación de una tesis de $\mathrm{T}$ no depende de si la gente observa si se producen tales o cuales combustiones, sino de la calidad del argumento en favor de dicha tesis. Ese encadenamiento de aseveraciones mezcla verdades con falsedades, y delata una concepción equivocada sobre la justificación de las teorías empíricas. Aunque T no trate sobre las posibles observaciones, cuando intentamos contrastar $\mathrm{T}$ lo hacemos auxiliados por fragmentos relativamente simples de otras teorías, incluyendo proposiciones poco controvertidas de teorías perceptivas. En ese sentido, T implica consecuencias sobre reacciones químicas, y - en conjunción con supuestos sencillos sobre nuestra percepción - T implica también consecuencias sobre posibles observaciones de reacciones químicas. Naturalmente, la calidad de un argumento - en el sentido relevante para evaluar si su conclusión está apropiadamente justificada- dependerá, entre otras cosas, de la justificación de sus premisas. La calidad de un argumento en favor de una tesis de T sí puede depender de si la gente observa esto o lo otro.

Quizá Deutsch rechazaría esa extrapolación y no querría suscribir respecto a una teoría química tesis análogas a las que suscribe respecto a una teoría de la referencia. La razón podría estar vinculada a cierto elemento diferencial. Hay bastante aceptación general de que la contrastación de hechos postulados en ámbitos empíricos como la química o la geo- 
logía depende de observaciones; con frecuencia, hay también concordancia sobre cuándo una determinada observación apoya tal o cual hecho. En la filosofía -o en las matemáticas- es muchísimo más controvertido qué papel desempeña la intuición en la justificación de una hipótesis. Es una diferencia cuantitativa importante. Pero si un filósofo insiste en que la contribución de nuestras intuiciones a la justificación de una teoría de la referencia es nula (motivado o no por ciertas opiniones escépticas sobre las intuiciones), un filósofo podría insistir en que la contribución de nuestras observaciones a la justificación de una teoría química es nula (motivado o no por ciertas opiniones escépticas sobre las observaciones; con el mismo tipo de legimitidad; no con la misma legitimidad: comprendemos mucho mejor cómo las observaciones apoyan las teorías empíricas que cómo las intuiciones apoyan las teorías filosóficas o matemáticas). Curiosamente, Deutsch parece congeniar con la postulación de intuiciones y con la justificación a priori (cf. pp. 458-460). Siendo así, es difícil entender cómo establecería la diferencia relevante que bloqueara la extrapolación.

\section{Capacidades expertas}

La tesis (M2), según la cual las supuestas intuiciones anti-descriptivistas puestas de relieve en casos del tipo Gödel-Schmidt no son suficientemente generales (para constituir indicios contrarios al descriptivismo), tiene cierta ambigüedad, o - al menos - una doble lectura según pongamos o no el acento en la parte señalada entre paréntesis. En las secciones 3, 4 y 6 he señalado problemas de inconsistencia y de ambigüedad en los diseños experimentales de Machery et al. Basándome en esos problemas, he negado que esos autores hayan ofrecido una apropiada justificación de la tesis (M2), incluso bajo la lectura meramente descriptivoestadística de (M2). En otras palabras: no han conseguido demostrar que la mayoría de sujetos asiáticos encuestados tengan las intuiciones descriptivistas que ellos les atribuyen. En las secciones 7 y 8 , he comentado algunos aspectos de la relación entre (M2) - en ese sentido descriptivo-estadístico- y la tesis (M1), según la cual casos del tipo Gödel-Schmidt no respaldan el anti-descriptivismo.

Conviene hacer explícito ya el otro punto débil de la posición de Machery et al. Supongamos que sí hubieran demostrado que esos sujetos tienen mayoritariamente intuiciones descriptivistas. Incluso así, no habrían proporcionado una apropiada justificación de (M2), si tenemos en cuenta las palabras entre paréntesis. Por consiguiente, no habrían justificado la tesis (M1). Veamos por qué.

Todo el diseño experimental de Machery et al. depende de un presupuesto metodológico muy controvertido. Ellos intentan medir qué respaldo tienen el descriptivismo y el anti-descriptivismo a partir de las intuiciones, y para esa tarea asumen que todas las intuiciones son igualmente válidas, que cada intuición tiene el mismo peso. En otras palabras, asumen que no existen capacidades expertas respecto a las intuiciones, que no hay posibilidad de educar-perfeccionar-refinar las intuiciones. Sin embargo, como he anticipado en la sección 7, la mejor teorización sobre la relación de justificación entre intuiciones y tesis filosóficas no necesariamente debe sostener que todas las intuiciones valen igual. ${ }^{13}$

${ }^{13}$ Ludwig (2007) ha enfatizado este grave inconveniente de las propuestas de Machery et al. 
Sería difícil negar que existen capacidades expertas respecto a la percepción sensorial. Aunque dos personas estén observando lo mismo, una puede detectar visualmente elementos que pasen desapercibidos a la otra. Mirando una radiografia, un traumatólogo o un dentista es capaz de reconocer lesiones que no reconocerá el neófito. Mediante el gusto y el olfato, un sumiller distingue matices que otras personas no podrían distinguir. Los ejemplos se multiplicarían. Conforme a esos datos, la mejor teorización sobre la relación de justificación entre las observaciones y las tesis empíricas implica que no todas las observaciones valen igual; los juicios de los sujetos basados en sus observaciones no son equiparables; algunas observaciones, y los juicios basados en ellas, serán más fiables que otras. Como mínimo, es un supuesto muy cuestionable que no suceda algo análogo respecto a la conexión entre intuiciones y tesis filosóficas. ${ }^{14}$

Un filósofo que admita la existencia de capacidades expertas respecto a la observación quizá rechace la posición análoga respecto a la intuición por razones emparentadas con lo ya indicado al final de la sección anterior. Nuestra mayor comprensión de la observación incluiría el manejo de ciertas hipótesis sobre qué tipo de «entrenamiento» recibe un dentista o un traumatólogo, en virtud del cual queda habilitado como alguien comparativamente mejor capacitado en (algunas de) sus observaciones. Las hipótesis sobre qué tipo de «entrenamiento» recibe un filósofo para educar-perfeccionar-refinar sus intuiciones serían bastante más inestables y controvertidas. Con todo, no veo en los textos de Machery et al. ninguna justificación — ni empírica ni argumentativa-conceptual - para rechazar que haya capacidades expertas respecto a la intuición.

\section{REFERENCIAS}

Beebee, James y Undercoffer, Ryan. 2016. Individual and Cross-Cultural Differences in Semantic Intuitions: New Experimental Findings. Journal of Cognition and Culture 16.

Deutsch, Max. 2009. Experimental Philosophy and the Theory of Reference. Mind and Language 24: 445466.

Grice, H. Paul. 1975. Logic and Conversation. En H. P. Grice, Studies in the Ways of Words, Cambridge, Mass.: Harvard University Press, 1989, pp. 22-40. Publicado originalmente en 1975.

Hazlett, Allan. 2010. The Myth of Factive Verbs. Philosophy and Phenomenological Research 80: 497-522.

Ichikawa, Jonathan, Maitra, Ishani y Weatherson, Brian. 2012. In Defense of a Kripkean Dogma. Philosophy and Phenomenological Research 82: 56-68.

Kallestrup, Jesper. 2016. Counteractuals, Counterfactuals and Semantic Intuitions. Review of Philosophy and Psychology 7:35-54.

Kripke, Saul. 1972/1980. Naming and Necessity. En D. Davidson y G. Harman (eds.), Semantics of Natural Language, Dordrecht: D. Reidel, 1972, pp. 253-355 y 763-769. Reimpresión con un prefacio añadido: Harvard: Harvard University Press, 1980.

${ }^{14}$ Machery et al. (2004, B8-B9) hacen una alusión a esta cuestión. Pero no presentan justificación alguna en favor de dicho supuesto. Sólo acusan de narcisismo a quien pretenda que las intuiciones de los «filósofos académicos occidentales» son más fiables que las intuiciones contrarias de otros grupos culturales o lingüísticos. La etiqueta 'occidental' está fuera de lugar. Que (algunos de) los juicios basados en observaciones de un traumatólogo sean más fiables que los de un neófito no es narcisista, ni implica que (algunos de) los juicios basados en observaciones de los traumatólogos occidentales sean más fiables que los juicios contrarios basados en observaciones de otros grupos culturales o lingüísticos. 
Kripke, Saul. 1977. Speaker's Reference and Semantic Reference. En P. French, T. Uehling y H. Wettstein (eds.), Contemporary Perspectives in the Philosophy of Language, Minneapolis: University of Minnesota Press, pp. 6-27.

Lam, Barry. 2010. Are Cantonese-speakers really descriptivists? Revisiting cross-cultural semantics. Cognition 115: 320-329.

Ludwig, Kirk. 2007. The Epistemology of Thought Experiments: First Person versus Third Person Approaches. Midwest Studies in Philosophy 31: 128-159.

Machery, Edouard, Mallon, Ron, Nichols, Shaun y Stich, Sthephen. 2004. Semantics, Cross-Cultural Style. Cognition 92: B1-B12.

Machery, Edouard, Sytsma, Justin y Deutsch, Max. 2015. Speaker's Reference and Cross-Cultural Semantics. En A. Bianchi (ed.), On Reference, Oxford: Oxford University Press, pp. 62-76.

Martí, Genoveva. 2009. Against Semantic Multi-Culturalism. Analysis 69: 42-48.

Pérez Otero, Manuel. 2006. Esbozo de la filosofía de Kripke. Barcelona: Editorial Montesinos.

Pérez Otero, Manuel. 2007. An Evaluation of Kripke's Account of the Illusion of Contingency. Critica. Revista Hispanoamericana de Filosofía 39: 19-44.

Pérez Otero, Manuel. 2013. Kripke: reorientaciones en el giro lingüístico-representacional. En D. P. Chico (coordinador) Perspectivas en la filosofía del lenguaje. Vol. I, Zaragoza: Prensas Universitarias de Zaragoza, pp. 242-272.

Sytsma, Justin y Livengood, Jonathan. 2011. A new perspective concerning experiments on semantic intuitions. Australasian Journal of Philosophy 89: 315-332.

Manuel Pérez Otero (Doctorado en Filosofía, 1996) es Profesor Titular en el Departamento de Filosofía de la Universidad de Barcelona, y miembro de LOGOS (Research Group in Analytic Philosophy) desde su fundación. Investiga preferentemente en epistemología, filosofía del lenguaje, lógica filosófica y metafísica. Sobre esas temáticas ha publicado varios libros y artículos en revistas especializadas como Crítica, Dialectica, Disputatio, European Review of Philosophy, Grazer Philosophische Studien, History and Philosophy of Logic, Philosophical Studies, Philosophical Papers, Philosophy and Phenomenological Research, Synthese, Theoria y Teorema.

Dirección: Departamento de Filosofía. Univ. de Barcelona. C/ Montalegre 6. 08001 Barcelona. Email: perez.otero@ub.edu 